\title{
Bargaining and Price-of-Anarchy in Repeated Inter-Session Network Coding Games
}

\author{
Amir-Hamed Mohsenian-Rad ${ }^{\dagger}$, Jianwei Huang ${ }^{\ddagger}$, Vincent W.S. Wong ${ }^{\dagger}$, Robert Schober ${ }^{\dagger}$ \\ ${ }^{\dagger}$ Department of Electrical and Computer Engineering, University of British Columbia, Vancouver, Canada \\ ${ }_{\ddagger}^{\ddagger}$ Information Engineering Department, Chinese University of Hong Kong, Hong Kong, China \\ e-mails: \{hamed, vincentw, rschober\}@ece.ubc.ca, jwhuang@ie.cuhk.edu.hk
}

\begin{abstract}
Most of the previous work on network coding has assumed that the users are not selfish and always follow the designed coding schemes. However, recent results have shown that selfish users do not have the incentive to participate in inter-session network coding in a static non-cooperative game setting. As a result, the worst-case network efficiency (i.e., the price-of-anarchy) can be as low as $22 \%$. In this paper, we show that if the same game is played repeatedly, then the price-ofanarchy can be significantly improved to $48 \%$. We propose a grim-trigger strategy that encourages users to cooperate and participate in the inter-session network coding. A key challenge is to determine a common cooperative coding rate that the users should mutually agree on. We propose to resolve the conflict of interest among the users through a bargaining process. We derive a tight upper bound for the price-of-anarchy which is valid for any bargaining scheme. Moreover, we propose a simple and efficient min-max bargaining solution that can achieve this upper bound. Our results represent one of the first steps towards designing practical inter-session network coding schemes that can achieve reasonable performance for selfish users.
\end{abstract}

\section{INTRODUCTION}

Since the seminal paper by Ahlswede et al. [1], a rich body of work has been reported on how network coding can improve performance in both wired and wireless networks [2]-[4]. In general, network coding is performed by encoding multiple packets either from the same user or from different users. The former is called intra-session network coding [1], [2], while the latter is called inter-session network coding [3], [4]. A common assumption in most existing network coding schemes is that the users are cooperative and do not pursue their own interests. However, this assumption can be violated in practice.

In non-cooperative network coding, each user individually decides on whether to use network coding and how to use it to maximize its own payoff. If the coding is across different unicast users as in inter-session network coding, then the coding users rely on each other as they need to receive some remedy packets to decode the coded data at their destinations.

Recent results in [5]-[7] have shown that if the inter-session network coding game is played only once (i.e., as a static game [8]), then users do not have the incentive to provide each other with the needed remedy packets. In that case, the Nash equilibrium is not to perform network coding. The price-ofanarchy (PoA), i.e., the worst-case efficiency compared with the optimal network performance, can be as low as $22 \%$ [6].

In this paper, we study the more realistic scenario where the inter-session network coding game in [6] is likely to be played repeatedly (i.e., the game is not static any more). This can be interpreted as a network setting where users have many packets to transmit. As the users continue sending more packets, they can take into account the history of the game (e.g., whether or not the other users have provided the needed remedy packets in the past), and plan their future actions accordingly.

It is usually expected that a repeated game encourages cooperation among users [8], [9]. However, the key challenge in a repeated inter-session network coding game is that cooperation is not well-defined. It is not immediately clear for the users how they should cooperate. This introduces a bargaining problem among the users to search for a mutually acceptable network coding rate, before they start data transmission. We show that a good bargaining solution along with a grim-trigger strategy can be used to encourage cooperation and facilitate inter-session network coding. We also analyze the general properties of all possible bargaining schemes and provide an upper bound on the PoA which is valid for any bargaining scheme. We show that the PoA in the repeated two-user game can be improved to up to $48 \%$. This upper bound can be reached by using a min-max bargaining scheme. The key contributions of this paper can be summarized as follows:

- New Formulation: To the best of our knowledge, we are the first to formulate non-cooperative inter-session network coding as a repeated resource allocation game.

- Equilibrium Strategy Design: We show that a grim-trigger strategy can form a subgame perfect equilibrium for the repeated inter-session network coding game as long as the network coding users can agree on using a certain intersession network coding rate. Reaching such an agreement is non-trivial in general. It involves solving a bargaining problem that resolves the conflict of interest among users.

- Performance Bounds for All Bargaining Schemes: We characterize the general properties of all possible bargaining schemes and show that, for any bargaining scheme, the PoA of the two-user repeated inter-session network coding game is upper-bounded by $48 \%$.

- Simple and Efficient Bargaining Solution: We propose a novel min-max bargaining scheme to reach an efficient agreement among users. Numerical results show that the PoA reaches its upper bound for the widely used class of $\alpha$-fair utility functions if this bargaining scheme is used.

The results in this paper are different from the existing results on network coding games (e.g., in [5]-[7], [10]-[12]). The studies in [10], [11] focus on intra-session network coding, while here we address inter-session network coding. Similar to [7], we study inter-session network coding in a 


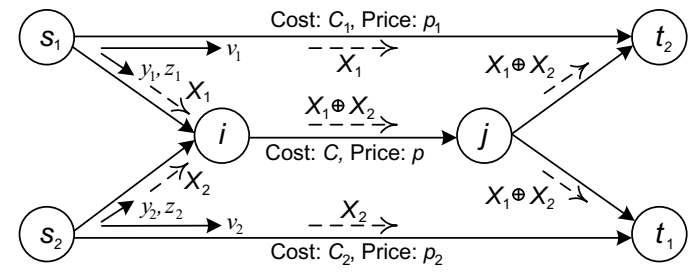

Fig. 1. A butterfly network with two unicast sessions, called users. They can perform inter-session network coding over links $(i, j),\left(s_{1}, t_{2}\right)$, and $\left(s_{2}, t_{1}\right)$. Packet $X_{1} \oplus X_{2}$ is obtained by joint encoding of packets $X_{1}$ and $X_{2}$.

butterfly topology. However, we further investigate the impacts of the utility functions of the users, the cost of the links, and the PoA. Finally, we study repeated games, while the results in [5]-[7], [10]-[12] are for static network coding games.

The rest of this paper is organized as follows. In Section II, we introduce the system model and review the static game results in [6]. The repeated game is formulated in Section III. Our results on subgame perfect equilibrium, bargaining, and the PoA bound are given in Section IV. The min-max bargaining solution and its resulting PoA are discussed in Section V. The paper is concluded in Section VI.

\section{System Model And BACKGROUND}

Consider the network in Fig. 1, which is referred to as a butterfly network in the network coding literature ${ }^{1}$. It consists of two end-to-end users. The bottleneck link $(i, j)$ is shared by both users $\mathcal{N}=\{1,2\}$. For each user $n \in \mathcal{N}$, the source and the destination nodes are denoted by $s_{n}$ and $t_{n}$, respectively. The two direct side links $\left(s_{1}, t_{2}\right)$ and $\left(s_{2}, t_{1}\right)$ allow the users to perform inter-session network coding as we explain next.

Users 1 and 2 can mark their packets (e.g., by setting a single-bit flag in the packet header) for either routing or network coding. At node $i$, all packets that are marked for routing are simply forwarded to node $j$ through link $(i, j)$. However, the packets that are marked for network coding are treated differently. Let $X_{1}$ and $X_{2}$ denote two packets which are marked for network coding and are sent from nodes $s_{1}$ and $s_{2}$ to node $i$, respectively. Node $i$ can encode packets $X_{1}$ and $X_{2}$ (e.g., using XOR encoding [13]), and send the resulting packet, denoted by $X_{1} \oplus X_{2}$, towards node $j$ (and from there to $t_{1}$ and $t_{2}$ ). Given the remedy data $X_{1}$ from the side link $\left(s_{1}, t_{2}\right)$ and the remedy data $X_{2}$ from the side link $\left(s_{2}, t_{1}\right)$, nodes $t_{2}$ and $t_{1}$ can decode their own data packets $X_{2}$ and $X_{1}$ based on the encoded packets they receive from node $j$. Clearly, the benefit of network coding is to reduce the traffic on bottleneck link $(i, j)$ by sending only one packet $X_{1} \oplus X_{2}$, rather than two packets $X_{1}$ and $X_{2}$, over this link.

We define the following notations for data rates in Fig. 1:

- $y_{n}$ : Transmission rate of sending routing packets from node $s_{n}$ to node $i$, for each user $n \in \mathcal{N}$.

- $z_{n}$ : Transmission rate of sending network coding packets from node $s_{n}$ to node $i$, for each user $n \in \mathcal{N}$.

- $v_{n}$ : Transmission rate of sending remedy packets from node $s_{n}$ over its side link, for each user $n \in \mathcal{N}$.

${ }^{1}$ Although the network coding scenario in Fig. 1 is simple, it can be used as a building block for more general scenarios. For example, in [2], [3] the network is modeled as a superposition of several butterfly networks. Thus, understanding this simple model is a key to understand more general networks.
In this paper, we make the following key assumption.

Assumption 1: The users are autonomous and have control over their own transmission rates. They individually indicate, via marking, whether their packets should participate in intersession network coding or simply be forwarded over link $(i, j)$.

In this setting, the intermediate node $i$ encodes packets at rate $\min \left\{z_{1}, z_{2}\right\}$ and forwards the rest of the packets, without encoding their contents, at rate $y_{1}+y_{2}+\left|z_{1}-z_{2}\right|$. Thus, the total rate on bottleneck link $(i, j)$ becomes $\max \left\{z_{1}, z_{2}\right\}$. As a result, nodes $t_{1}$ and $t_{2}$ receive information at rates $y_{1}+$ $\min \left\{z_{1}, v_{2}\right\}$ and $y_{2}+\min \left\{z_{2}, v_{1}\right\}$, respectively [6].

\section{A. Utility, Cost, and Price Functions}

We assume that each user $n \in \mathcal{N}$ has a utility function $U_{n}$, representing its benefit from its achieved data rate. On the other hand, link $(i, j)$ has a cost function $C$, which depends on its total traffic load $y_{1}+y_{2}+\max \left\{z_{1}, z_{2}\right\}$. Similarly, links $\left(s_{1}, t_{2}\right)$ and $\left(s_{2}, t_{1}\right)$ have cost functions $C_{1}$ and $C_{2}$, which depend on their load, i.e., $v_{1}$ and $v_{2}$, respectively.

Assumption 2: The utility functions $U_{1}$ and $U_{2}$ are concave, non-negative, increasing, and differentiable [14].

Assumption 3: The link cost functions are given as

$$
C(q)=\frac{a}{2} q^{2}, \quad C_{1}(q)=\frac{b_{1}}{2} q^{2}, \quad C_{2}(q)=\frac{b_{2}}{2} q^{2}, \quad \forall q \geq 0,
$$

where $a>0, b_{1}>0$, and $b_{2}>0$. The convex quadratic cost functions in (1) are related to linear price functions $p(q)=a q$, $p_{1}(q)=b_{1} q$, and $p_{2}(q)=b_{2} q$. In fact, $C(q)=\int_{0}^{q} p(\theta) d \theta$, $C_{1}(q)=\int_{0}^{q} p_{1}(\theta) d \theta$, and $C_{2}(q)=\int_{0}^{q} p_{2}(\theta) d \theta$.

Quadratic cost and linear price functions are the only cost and price functions that satisfy the four axioms of rescaling, consistency, positivity, and additivity in cost-sharing systems [15]. They are often used in network resource management (cf. [16], [17]) to model the queueing delay on each link.

\section{B. Optimization-Based Resource Allocation}

Let $\boldsymbol{y}=\left(y_{1}, y_{2}\right), \boldsymbol{v}=\left(v_{1}, v_{2}\right)$, and $\boldsymbol{z}=\left(z_{1}, z_{2}\right)$. The network aggregate surplus is defined as the total utility achieved by the users minus the total cost of the links:

$$
\begin{aligned}
\mathbb{S}(\boldsymbol{y}, \boldsymbol{z}, \boldsymbol{v}) & =U_{1}\left(y_{1}+\min \left\{z_{1}, v_{2}\right\}\right) \\
& +U_{2}\left(y_{2}+\min \left\{z_{2}, v_{1}\right\}\right)-C_{1}\left(v_{1}\right) \\
& -C_{2}\left(v_{2}\right)-C\left(y_{1}+y_{2}+\max \left\{z_{1}, z_{2}\right\}\right) .
\end{aligned}
$$

Given complete knowledge and centralized control of the network in Fig. 1, an efficient rate allocation is obtained by solving the following optimization problem [16], [17].

\section{Problem 1 (Network Surplus Maximization Problem):}

$$
\begin{array}{ll}
\underset{\boldsymbol{y}, \boldsymbol{z}, \boldsymbol{v}}{\operatorname{maximize}} & \mathbb{S}(\boldsymbol{y}, \boldsymbol{z}, \boldsymbol{v}) \\
\text { subject to } & y_{1}, y_{2}, z_{1}, z_{2}, v_{1}, v_{2} \geq 0 .
\end{array}
$$

Let $\boldsymbol{y}^{S}=\left(y_{1}^{S}, y_{2}^{S}\right), \boldsymbol{v}^{S}=\left(v_{1}^{S}, v_{2}^{S}\right)$, and $\boldsymbol{z}^{S}=\left(z_{1}^{S}, z_{2}^{S}\right)$ be an optimal solution for Problem 1 . We can verify that $v_{1}^{S}=z_{1}^{S}$ $=v_{2}^{S}=z_{2}^{S}$, i.e., the network coding users send the coded and remedy packets at the same rate in an optimal rate allocation. 


\section{Pricing and Resource Allocation Game}

If the network has no centralized controller and Assumption 1 holds, pricing can be used to encourage efficient resource allocation in a distributed fashion [14]. Given the rate vectors $\boldsymbol{y}$ and $\boldsymbol{z}$ from the users, the shared link $(i, j)$ can set a price

$$
\mu(\boldsymbol{y}, \boldsymbol{z})=p\left(y_{1}+y_{2}+\max \left\{z_{1}, z_{2}\right\}\right)
$$

for any uncoded data rate it carries, where price function $p(q)$ is described in Assumption 3. It can set a separate price

$$
\sigma(\boldsymbol{y}, \boldsymbol{z})=\beta \mu(\boldsymbol{y}, \boldsymbol{z})=\beta p\left(y_{1}+y_{2}+\max \left\{z_{1}, z_{2}\right\}\right)
$$

for any coded data it carries. Here, $\beta \in(0,1]$ is the price discrimination parameter. Note that only the choice of $\beta=\frac{1}{2}$ can avoid over- or under-charging of network coding users [6].

Assumption 4: Throughout this paper, we set $\beta=\frac{1}{2}$.

On the other hand, given data rates $\boldsymbol{v}$ for the remedy packets, the side links $\left(s_{1}, t_{2}\right)$ and $\left(s_{2}, t_{1}\right)$ set their prices as

$$
\mu_{1}\left(v_{1}\right)=p_{1}\left(v_{1}\right) \quad \text { and } \quad \mu_{2}\left(v_{2}\right)=p_{2}\left(v_{2}\right),
$$

for any data rate they carry. Users are charged as follows:

- User 1 pays

$$
\begin{aligned}
& \sigma(\boldsymbol{y}, \boldsymbol{z}) \min \left(z_{1}, z_{2}\right)+\mu(\boldsymbol{y}, \boldsymbol{z})\left(z_{1}-\min \left(z_{1}, z_{2}\right)\right) \\
& +\mu(\boldsymbol{y}, \boldsymbol{z}) y_{1}=\mu(\boldsymbol{y}, \boldsymbol{z})\left(y_{1}+z_{1}-(1-\beta) \min \left\{z_{1}, z_{2}\right\}\right)
\end{aligned}
$$

to link $(i, j)$ and $v_{1} \mu_{1}\left(v_{1}\right)$ to link $\left(s_{1}, t_{2}\right)$.

- User 2 pays to links $(i, j)$ and $\left(s_{2}, t_{1}\right)$ similarly.

The users then select their rates to maximize their own surplus, i.e., utility minus charges [16], [17]. Clearly, each user's surplus also depends on the data rates selected by other users, leading to a resource allocation game among all users:

\section{Game 1 (Non-cooperative Resource Allocation Game):}

- Players: Users in set $\mathcal{N}$.

- Strategies: Transmission rates $\boldsymbol{y}, \boldsymbol{z}$, and $\boldsymbol{v}$.

- Payoffs: $P_{n}(\cdot)$ for each user $n \in \mathcal{N}$, where

$$
\begin{aligned}
& P_{1}\left(y_{1}, z_{1}, v_{1} ; y_{2}, z_{2}, v_{2}\right)=U_{1}\left(y_{1}+\min \left\{z_{1}, v_{2}\right\}\right) \\
& \quad-v_{1} p_{1}\left(v_{1}\right)-\left(y_{1}+z_{1}-(1-\beta) \min \left\{z_{1}, z_{2}\right\}\right) \\
& \quad \times p\left(y_{1}+y_{2}+\max \left\{z_{1}, z_{2}\right\}\right), \\
& P_{2}\left(y_{2}, z_{2}, v_{2} ; y_{1}, z_{1}, v_{1}\right)=U_{2}\left(y_{2}+\min \left\{z_{2}, v_{1}\right\}\right) \\
& \quad-v_{2} p_{2}\left(v_{2}\right)-\left(y_{2}+z_{2}-(1-\beta) \min \left\{z_{1}, z_{2}\right\}\right) \\
& \quad \times p\left(y_{1}+y_{2}+\max \left\{z_{1}, z_{2}\right\}\right) .
\end{aligned}
$$

Game 1 is a static game and is played only once. The repeated version of Game 1 will be formulated in Section III.

\section{Efficiency and Price-of-Anarchy of Game 1}

The selfish nature of the players in Game 1 leads to undesirable and inefficient network performance. To see this, we first introduce the following definitions.

Definition 1 (Nash equilibrium): The non-negative rates $\boldsymbol{y}^{*}$ $=\left(y_{1}^{*}, y_{2}^{*}\right), \boldsymbol{v}^{*}=\left(v_{1}^{*}, v_{2}^{*}\right)$, and $\boldsymbol{z}^{*}=\left(z_{1}^{*}, z_{2}^{*}\right)$ form a Nash equilibrium of Game 1 if no user $n \in \mathcal{N}$ can increase its payoff $P_{n}(\cdot)$ by unilaterally changing its own transmission rates. The Nash equilibrium predicts how Game 1 will be played.
Definition 2 (Efficiency): For a certain choice of system parameters, the efficiency at Nash equilibrium $\left(\boldsymbol{y}^{*}, \boldsymbol{v}^{*}, \boldsymbol{z}^{*}\right)$ is the ratio of the achieved network aggregate surplus $\mathbb{S}\left(\boldsymbol{y}^{*}, \boldsymbol{z}^{*}, \boldsymbol{v}^{*}\right)$ to the optimal network aggregate surplus $\mathbb{S}\left(\boldsymbol{y}^{S}, \boldsymbol{z}^{S}, \boldsymbol{v}^{S}\right)$.

Definition 3 (Price-of-anarchy): The price-of-anarchy, denoted by $\operatorname{PoA}($ Game 1, Problem 1), is the worst-case efficiency of a Nash equilibrium among all possible choices of parameters: the number of users, the utility, cost, and price functions.

Next, we notice that payoffs $P_{1}(\cdot)$ and $P_{2}(\cdot)$ are decreasing in $v_{1}$ and $v_{2}$, respectively. Thus, selfish network coding users 1 and 2 will always choose $v_{1}=0$ and $v_{2}=0$ to avoid payments over links $\left(s_{1}, t_{2}\right)$ and $\left(s_{2}, t_{1}\right)$, respectively. Being aware of this issue, users 1 and 2 will not participate in network coding as they cannot decode the encoded packets they receive. The following results are from [6, Theorem 11].

Theorem 1: (a) Game 1 has a unique Nash equilibrium.

(b) At Nash equilibrium of Game 1, we have

$$
v_{1}^{*}=z_{1}^{*}=0 \quad \text { and } \quad v_{2}^{*}=z_{2}^{*}=0 .
$$

(c) We have PoA (Game 1, Problem 1) $=\frac{2}{9} \approx 22 \%$.

The results in Theorem 1 are disappointing. In fact, we may compare the PoA results in Theorem 1 with the results in [16], where the PoA is at least $67 \%$ for a similar resource allocation game but with routing users. The results in Theorem 1 imply that although inter-session network coding can potentially improve network performance, it is sensitive to selfish behavior. The key contribution of this paper is to show that it is possible to design better strategies such that the PoA results can be improved when Game 1 is played repeatedly.

\section{Repeated InTER-SEssion Network Coding GAME}

Consider the case when Game 1 is played repeatedly. That is, after each time the users in $\mathcal{N}$ play Game 1 , it is likely that they play Game 1 again with a probability $\delta$. Parameter $\delta$ is called the discount factor [8]. Each time that Game 1 is played is referred to as one stage of the repeated game. A repeated game occurs if users have many packets to transmit.

If Game 1 is played multiple times, then the strategy space of the users will include their data rates at each stage of the game. Let $\boldsymbol{y}^{k}=\left(y_{1}^{k}, y_{2}^{k}\right), \boldsymbol{z}^{k}=\left(z_{1}^{k}, z_{2}^{k}\right)$, and $\boldsymbol{v}^{k}=\left(v_{1}^{k}, v_{2}^{k}\right)$ denote the actions played by the users at stage $k$, where $k \geq 1$. At the beginning of stage $k$, the data rates that have been already selected in stages $1, \ldots, k-1$ form the history of the game, while the data rates to be selected in stages $k, k+1, \ldots$ are the strategies of the users. For notational simplicity, for each $1 \leq l \leq m$, we define

$$
\mathcal{R}_{t=l}^{m}=\left\{\boldsymbol{y}^{t}, \boldsymbol{z}^{t}, \boldsymbol{v}^{t}\right\}_{t=l}^{m} .
$$

In this regard, $\mathcal{R}_{t=1}^{k-1}$ denotes the history and $\mathcal{R}_{t=k}^{\infty}$ denotes the strategies of the users, at each $k \geq 1$.

\section{Game 2 (Repeated Game 1):}

- Players: Users in set $\mathcal{N}$.

- Histories: Data rates $\mathcal{R}_{t=1}^{k-1}$, at each stage $k \geq 1$.

- Strategies: Contingency plans for selection of rates $\mathcal{R}_{t=k}^{\infty}$ at each stage $k \geq 1$ for any given history profile $\mathcal{R}_{t=1}^{k-1}$. 
- Payoffs: $Q_{n}(\cdot)$ for each user $n \in \mathcal{N}$, where at each $k \geq 1$,

$$
Q_{n}\left(\mathcal{R}_{t=k}^{\infty} \mid \mathcal{R}_{t=1}^{k-1}\right)=\sum_{t=k}^{\infty}(\delta)^{t-k} P_{n}\left(\boldsymbol{y}^{t}, \boldsymbol{z}^{t}, \boldsymbol{v}^{t}\right) .
$$

In Game 2, the single-stage payoffs $P_{1}(\cdot)$ and $P_{2}(\cdot)$ are as in Game 1. Then, payoffs $Q_{1}(\cdot)$ and $Q_{2}(\cdot)$ are defined as the discounted summations of the users' payoffs at each remaining stage of the game. Being at the beginning of stage $k \geq 1$, each user $n \in \mathcal{N}$ plans for its future data rates to maximize the expected payoff $Q_{n}(\cdot)$ it obtains during the rest of Game 2.

Definition 4 (Subgame): Given a history profile $\mathcal{R}_{t=1}^{k-1}$ at stage $k \geq 1$ of Game 2 , the rest of the repeated game at stages $k, k+1, \ldots$ is defined as a subgame at stage $k$.

The solution concept for a repeated game is the subgame perfect equilibrium which is defined as follows [8]:

Definition 5 (Subgame Perfect Equilibrium): A strategy profile $\mathcal{R}_{k=1}^{\infty}$ is a subgame perfect equilibrium of Game 2 if at any stage $k \geq 1$, the restricted strategy profile $\mathcal{R}_{t=k}^{\infty}$ is a Nash equilibrium for any subgame at stage $k$, formed by every given history $\mathcal{R}_{t=1}^{k-1}$. That is, at any stage and for any history profile, no user $n \in \mathcal{N}$ can increase its payoff $Q_{n}(\cdot)$ by unilaterally changing its own data rates in future stages.

Definition 6 (Efficiency): The efficiency at subgame perfect equilibrium $\mathcal{R}_{k=1}^{\infty}$ is defined as the average efficiency among all stages of Game 2, where at each stage $k \geq 1$, the efficiency for rates $\left(\boldsymbol{y}^{k}, \boldsymbol{z}^{k}, \boldsymbol{v}^{k}\right)$ is defined according to Definition 2.

Definition 7 (Price-of-anarchy): The price-of-anarchy, denoted by PoA(Game 2, Problem 1), is the worst-case (i.e., the smallest) efficiency at a subgame perfect equilibrium of Game 2 among all possible choices of all system parameters.

\section{Punishment AND BARgaining In Inter- Session Network CODING}

In this section, we analyze repeated Game 2 and show the following: First, a simple grim-trigger strategy encourages users to cooperate. Second, if the two users cooperate, they will select the same network coding rates. Third, the common network coding rate can be acquired via bargaining.

\section{A. Punishment and Grim-trigger Strategy}

At the end of each stage of Game 2, user 1 is able to find out whether user 2 has cooperated (i.e., sent enough remedy packets such that user 1 can decode all received encoded packets) during the current stage. Thus, user 1 can punish user 2 in the next stage, if user 2 has cheated and has not sent all remedy packets. A similar statement is true for user 2 .

Users 1 and 2 may use various punishment strategies against a cheating user. For example, if user 2 cheats at stage $k-1$ of Game 2, then user 1 may select its data rates $\left(y_{1}^{k}, z_{1}^{k}, v_{1}^{k}\right)$ to minimize user 2's payoff in the next stage. Another option for user 1 is simply not to participate in network coding by setting $v_{1}^{k}=z_{1}^{k}=0$. Punishment strategies can be either limited scope, i.e., last for only a few stages, or unlimited scope, i.e., last until the game ends. In this paper, we only consider the case when the punishment is not to participate in network coding forever. We will show that this simple punishment strategy can fully prevent cheating. To start with, we show that if users decide to cooperate, they choose the same coding rate.
Theorem 2: Assume that users select data rates $\boldsymbol{y}^{k}, \boldsymbol{z}^{k}$, and $\boldsymbol{v}^{k}$ at stage $k \geq 1$ of repeated Game 2 and we have

$$
v_{1}^{k}=z_{1}^{k}>v_{2}^{k}=z_{2}^{k} .
$$

That is, neither user 1 nor user 2 cheat, but user 1 participates in network coding with a higher rate than user 2 . Then, user 1 can unilaterally switch to new rates $\left(\bar{y}_{1}^{k}, \bar{v}_{1}^{k}, \bar{z}_{1}^{k}\right)$ such that

$$
\bar{y}_{1}^{k}=y_{1}^{k}+\left(z_{1}^{k}-z_{2}^{k}\right), \quad \bar{v}_{1}^{k}=\bar{z}_{1}^{k}=v_{2}^{k}=z_{2}^{k},
$$

to strictly increase its own payoff at stage $k$, while keeping the payoff of user 2 unchanged at stage $k$. A similar statement is true for user 2 if $v_{1}^{k}=z_{1}^{k}<v_{2}^{k}=z_{2}^{k}$.

The proof of Theorem 2 is given in Appendix A. From Theorem 2, if users 1 and 2 do not plan to cheat and want to cooperate, they should choose the same network coding rates:

$$
z_{1}^{k}=v_{1}^{k}=z_{2}^{k}=v_{2}^{k}, \quad \forall k \geq 1 .
$$

The above can help us to predict how users behave if they cooperate. However, we still need to answer two questions:

1) Which common rate

$$
z_{1}^{k}=v_{1}^{k}=z_{2}^{k}=v_{2}^{k}=z \geq 0, \quad \forall k \geq 1,
$$

should network coding users 1 and 2 choose?

2) How do network coding users 1 and 2 enforce cooperation such that they both have the incentive to send remedy packets at the desired rate $z \geq 0$ ?

We will answer the second question first. The first question will be answered in Section IV-B when we discuss bargaining.

Next, we explain how the users behave at each stage $k \geq 1$ of Game 2 if (11) holds for a pre-determined $z \geq 0$. For the ease of exposition, we define a new static game which is derived from static Game 1 and is parameterized with $z$.

Game 3 (Reduced Game 1 for a Given $z \geq 0$ ):

- Players: Users in set $\mathcal{N}$.

- Strategies: Transmission rates $\boldsymbol{y}$, when the network coding rates $\boldsymbol{v}$ and $\boldsymbol{z}$ are already set to

$$
z_{1}=v_{1}=z_{2}=v_{2}=z .
$$

- Payoffs: $P_{n}(\cdot)$ for each user $n \in \mathcal{N}$ as in Game 1.

Games 1 and 3 differ only due to (12). Since the coding rates are pre-determined, the strategy of the users in Game 3 is to only select the routing rates $\boldsymbol{y}$. From Theorem 1(a), Game 1 has a unique Nash equilibrium which depends on $z$.

Given $z \geq 0$, we denote the Nash equilibrium of Game 3 by $\boldsymbol{y}^{*}(z)$. Therefore, the payoff for each user $n \in \mathcal{N}$ at Nash equilibrium of Game 3 is denoted by

$$
P_{n}\left(\boldsymbol{y}^{*}(z), z_{1}=z_{2}=v_{1}=v_{2}=z\right) \text {. }
$$

For example, for network coding user 1, we have

$$
\begin{aligned}
P_{1}\left(\boldsymbol{y}^{*}(z), z\right)= & U_{1}\left(y_{1}^{*}(z)+z\right)-z p_{1}(z)-\left(y_{1}^{*}(z)+\beta z\right) \\
& \times p\left(y_{1}^{*}(z)+y_{2}^{*}(z)+z\right) .
\end{aligned}
$$

We now return to Game 2. Clearly, if the users agree on selecting their network coding rates according to (11), then at each stage $k \geq 1$, the users simply select their routing data rates to be $\bar{y}^{k}=\boldsymbol{y}^{*}(z)$. This helps us to introduce a 
strategy profile that can enforce cooperation, answering our second question posed earlier in this section.

Definition 8 (Grim-trigger Strategy): Given a coding rate $z \geq 0$, a grim-trigger strategy [8] for Game 2 is defined as

Step 1: Always participate in network coding according to the coding rate $z$ from the first stage of Game 2. Consequently, the users choose the routing rates according to the unique Nash equilibrium of Game 3 for the given $z$. That is, at stage $k$,

- User 1 sets $v_{1}^{k}=z_{1}^{k}=z$ and $y_{1}^{k}=y_{1}^{*}(z)$.

- User 2 sets $v_{2}^{k}=z_{2}^{k}=z$ and $y_{2}^{k}=y_{2}^{*}(z)$.

Go to Step 2 if user 1 or 2 deviate from coding rate $z$.

Step 2: Do not participate in network coding forever. That is, at each stage $k$,

- User 1 sets $v_{1}^{k}=z_{1}^{k}=0$ and $y_{1}^{k}=y_{1}^{*}(0)$.

- User 2 sets $v_{2}^{k}=z_{2}^{k}=0$ and $y_{2}^{k}=y_{2}^{*}(0)$.

Note that Step 2 is an unlimited scope punishment against the user that does not cooperate and deviates from the agreed coding rate $z$. We can show the following key result:

Theorem 3: Given a fixed $z \geq 0$, there exists a $\delta_{\min } \in(0,1]$ such that the grim-trigger strategy in Definition 8 forms a subgame perfect equilibrium for Game 2 if and only if

$$
\begin{aligned}
& P_{1}\left(\boldsymbol{y}^{*}(z), z\right) \geq P_{1}\left(\boldsymbol{y}^{*}(0), 0\right), \\
& P_{2}\left(\boldsymbol{y}^{*}(z), z\right) \geq P_{2}\left(\boldsymbol{y}^{*}(0), 0\right),
\end{aligned}
$$

and the discount factor $\delta$ satisfies $\delta_{\min } \leq \delta \leq 1$. We note that conditions (14) and (15) hold at least for coding rate $z=0$.

The proof of Theorem 3 is given in Appendix B. If (14) and (15) hold, it is beneficial for both users 1 and 2 to participate in network coding at rate $z$ as in Step 1, rather than not participating in network coding (i.e., choosing zero coding rate) as in Step 2. A larger discount factor $\delta$ implies that Game 2 is more likely to continue. Thus, the damage of future punishments due to deviating from coding rate $z$ is more significant, encouraging users to cooperate.

As an example, consider Fig. 1 and assume that

$$
\begin{gathered}
U_{1}(x)=\log (1+x), \quad U_{2}(x)=0.75 \log (1+x), \\
a=1, \quad b_{1}=0.5, \quad b_{2}=0.25, \quad \beta=0.5 .
\end{gathered}
$$

We can verify that if we select (according to the min-max bargaining scheme in Section V) $z=0.3$, then $y_{1}^{*}(z)=0.128$, $y_{2}^{*}(z)=0$, and at each stage of Game 2, the users play a game according to Table I, where the numbers indicate the payoffs for users 1 and 2, respectively. In this example, the grimtrigger strategy is a subgame perfect equilibrium and the users always cooperate if $\delta \geq \max \left\{\frac{0.24-0.19}{0.24-0.11}, \frac{0.14-0.12}{0.14-0.08}\right\} \approx 0.38$. It is interesting to note that the payoffs in Table I resemble those in the prisoner's dilemma game [8, p. 110]. It is wellknown that players have the incentive to cooperate if the prisoner's dilemma game is played repeatedly. However, the key difference between a repeated prisoner's dilemma game and Game 2 is that cooperation is not well-defined in Game 2 as it is not clear for the users which common/cooperative coding rate $z$ they should choose as we discuss next.
TABLE I

PAyOFFs AT EACH STAGE OF GAME 2 When (16) AND (17) HOLD.

\begin{tabular}{c|c|c|c|}
\multicolumn{3}{c}{ User 2} \\
\cline { 2 - 4 } User 1 & Strategy & Cooperate & Cheat \\
\cline { 2 - 4 } & Cooperate & $(0.19,0.12)$ & $(-0.08,0.14)$ \\
\cline { 2 - 4 } & Cheat & $(0.24,-0.10)$ & $(0.12,0.08)$ \\
\cline { 2 - 4 } & & \multicolumn{3}{|c}{}
\end{tabular}

\section{B. Bargaining}

So far, we have assumed that the common network coding rate $z \geq 0$ is given. In this section, we will discuss how the network coding users 1 and 2 can agree on the choice of $z$.

Clearly, if it was up to user 1 to decide, then it would selfishly select $z$ as the solution of the following problem

$$
\underset{z \geq 0}{\operatorname{maximize}} P_{1}\left(\boldsymbol{y}^{*}(z), z\right)
$$

to maximize its own payoff at each stage of Game 2. Similarly, user 2 would select $z$ as the solution of the following problem

$$
\underset{z \geq 0}{\operatorname{maximize}} P_{2}\left(\boldsymbol{y}^{*}(z), z\right) .
$$

However, in either case, there is no guarantee that (14) and (15) hold. Even if they do, the obtained $z$ may not be fair and mutually acceptable for both users. In that case, one of the users may refuse to use the grim-trigger strategy. The following issues need to be addressed in the current situation:

- There can be a conflict of interest between network coding users 1 and 2 for selection of data rate $z \geq 0$.

- No agreement on data rate $z \geq 0$ can be imposed on either user 1 or user 2 due to Assumption 1.

A natural way of dealing with these issues is to consider a bargaining problem in cooperative game theory [18], where two players negotiate on the details of cooperation.

A well-known bargaining solution is the Nash bargaining solution [19]. In the inter-session network coding context, it is obtained by solving the following optimization problem, where the objective function is the multiplication of the extra payoffs users 1 and 2 achieve at network coding rate $z \geq 0$ :

$$
\begin{array}{cl}
\underset{z \geq 0}{\operatorname{maximize}} & \left(P_{1}\left(\boldsymbol{y}^{*}(z), z\right)-P_{1}\left(\boldsymbol{y}^{*}(0), 0\right)\right) \\
& \times\left(P_{2}\left(\boldsymbol{y}^{*}(z), z\right)-P_{2}\left(\boldsymbol{y}^{*}(0), 0\right)\right) \\
\text { subject to } & P_{1}\left(\boldsymbol{y}^{*}(z), z\right) \geq P_{1}\left(\boldsymbol{y}^{*}(0), 0\right), \\
& P_{2}\left(\boldsymbol{y}^{*}(z), z\right) \geq P_{2}\left(\boldsymbol{y}^{*}(0), 0\right) .
\end{array}
$$

A Nash bargaining solution always exists and is unique [19]. It leads to a fair division of the cooperation benefit among the network coding users. A similar (but significantly easier to calculate) bargaining solution will be shown in Section V.

In general, for any choice of the bargaining solution, we can analyze the performance in terms of the achieved PoA (Game 2, Problem 1). We note that due to Theorem 3, the data rates of the users at all stages of Game 2 are set according to the rates in Step 1 of the grim-trigger strategy in Definition 8. Therefore, given the bargaining solution $z$, efficiency at every stage (thus for the whole repeated game) is obtained as the ratio of the network aggregate surplus $\mathbb{S}\left(\boldsymbol{y}^{*}(z), z_{1}=\right.$ $\left.z_{2}=v_{1}=v_{2}=z\right)$ to the optimal network aggregate surplus $\mathbb{S}\left(\boldsymbol{y}^{S}, \boldsymbol{z}^{S}, \boldsymbol{v}^{S}\right)$. Then, PoA (Game 2, Problem 1) for the considered bargaining scheme is the worst-case efficiency. 


\section{Upper Bound on Price-of-Anarchy of Game 2}

As a special case of Theorem 3, the following holds.

Corollary 1: If either

$$
P_{1}\left(\boldsymbol{y}^{*}(z), z\right)<P_{1}\left(\boldsymbol{y}^{*}(0), 0\right), \quad \forall z>0,
$$

or

$$
P_{2}\left(\boldsymbol{y}^{*}(z), z\right)<P_{2}\left(\boldsymbol{y}^{*}(0), 0\right), \quad \forall z>0,
$$

then the grim-trigger strategy in Definition 8 is a subgame perfect equilibrium if and only if the common coding rate is

$$
z=0
$$

for any value of discount factor $\delta \in(0,1]$. That is, no network coding is performed at the subgame perfect equilibrium.

Corollary 1 can help us find an upper bound for the PoA of Game 2, which holds for any bargaining scheme. In fact, for a scenario where either (21) or (22) holds, all possible bargaining schemes lead to the same bargaining solution as in (23). Therefore, any efficiency value that is obtained in this case will form an upper bound for PoA (Game 2, Problem 1).

If the coding rate is $z=0$ as in (23), Step 1 and Step 2 in the grim-trigger strategy will be identical and we have

$$
z_{1}^{k}=v_{1}^{k}=z_{2}^{k}=v_{2}^{k}=0, \quad \boldsymbol{y}^{k}=\boldsymbol{y}^{*}(0), \quad \forall k \geq 1 .
$$

That is, the users simply select the Nash equilibrium of the static Game 1 at every stage of the repeated Game 2 (see (6) in Theorem 1). This directly results in the next theorem.

Theorem 4: For a certain choice of system parameters such that either (21) or (22) hold, the efficiency at the subgame prefect equilibrium of repeated Game 2 is equal to the efficiency at Nash equilibrium of static Game 1.

It is shown in [6, Theorem 11] that the worst-case efficiency of static Game 1 occurs under the following conditions:

- The utility functions of the users are linear. That is,

$$
U_{n}(x)=\gamma_{n} x, \quad \forall n \in \mathcal{N} .
$$

- The cost parameters for side links are negligible. That is,

$$
b_{1} \rightarrow 0 \quad \text { and } \quad b_{2} \rightarrow 0 .
$$

The intuition behind (26) is clear: if the side links have low cost, then performing inter-session network coding can bring significant throughput gains to the users without major increase in the cost. Thus, not performing inter-session network coding in this case degrades the system performance the most.

From the above discussion on the efficiency of Game 1, together with Theorem 4 and Corollary 1, we can find tight upper bound for PoA (Game 2, Problem 1), if we can obtain the worst-case efficiency among all choices of parameters which satisfy (25) and (26), while (21) or (22) hold.

Lemma 1: Given an arbitrary $z \geq 0$, we can show that

(a) If $y_{1}^{*}(z)>0$ and $y_{2}^{*}(z)>0$, then

$$
\begin{aligned}
& U_{1}^{\prime}\left(y_{1}^{*}(z)+z\right)=a\left(y_{1}^{*}(z)+y_{2}^{*}(z)+(1+\beta) z\right)+a y_{1}^{*}(z) \\
& U_{2}^{\prime}\left(y_{2}^{*}(z)+z\right)=a\left(y_{1}^{*}(z)+y_{2}^{*}(z)+(1+\beta) z\right)+a y_{2}^{*}(z) .
\end{aligned}
$$

(b) If $y_{1}^{*}(z)>0$ and $y_{2}^{*}(z)=0$, then

$$
\begin{aligned}
& U_{1}^{\prime}\left(y_{1}^{*}(z)+z\right)=a\left(y_{1}^{*}(z)+(1+\beta) z\right)+a y_{1}^{*}(z), \\
& U_{2}^{\prime}(z) \leq a\left(y_{1}^{*}(z)+(1+\beta) z\right) .
\end{aligned}
$$

(c) If $y_{1}^{*}(z)=0$ and $y_{2}^{*}(z)=0$, then

$$
U_{1}^{\prime}(z) \leq a(1+\beta) z, \quad U_{2}^{\prime}(z) \leq a(1+\beta) z .
$$

We can verify Lemma 1 by checking the Karush-KuhnTucker (KKT) conditions. The case when $y_{1}^{*}(z)=0$ and $y_{2}^{*}(z)>0$ can be modeled similarly.

Proposition 1: Given a $z \geq 0$, if conditions (25) and (26) hold, then at Nash equilibrium of the reduced Game 3,

$$
\left[\begin{array}{l}
y_{1}^{*}(z) \\
y_{2}^{*}(z)
\end{array}\right]= \begin{cases}{\left[\frac{2 \gamma_{1}-\gamma_{2}-a(1+\beta) z}{3 a} \frac{2 \gamma_{2}-\gamma_{1}-a(1+\beta) z}{3 a}\right],} & 0 \leq z<\frac{2 \gamma_{2}-\gamma_{1}}{a(1+\beta)} \\
{\left[\begin{array}{ll}
\left.\frac{\gamma_{1}-a(1+\beta) z}{2 a}\right], & \frac{2 \gamma_{2}-\gamma_{1}}{a(1+\beta)} \leq z<\frac{\gamma_{1}}{a(1+\beta)} \\
0
\end{array}\right]} & \frac{\gamma_{1}}{a(1+\beta)} \leq z .\end{cases}
$$

Without loss of generality, here we assumed that $\gamma_{1} \geq \gamma_{2}$.

The proof of Proposition 1 is given in Appendix C. From Proposition 1, we can obtain closed-form expressions for $P_{1}\left(\boldsymbol{y}^{*}(z), z\right)$ and $P_{2}\left(\boldsymbol{y}^{*}(z), z\right)$ for any $z \geq 0$. Then, we can evaluate conditions (21) and (22) in Corollary 1 and determine whether users 1 and 2 can reach a non-zero bargaining solution by checking the three possible ranges of $z$ in Proposition 1 .

Theorem 5: Among all choices of parameters such that

- Condition 1: Both (25) and (26) hold

- Condition 2: Either (21) or (22) hold

the worst-case efficiency at the subgame perfect equilibrium of Game 2 becomes $\frac{12}{25}$ and occurs for

$$
a=1, \quad \gamma_{2}=\frac{\gamma_{1}}{4} .
$$

The proof of Theorem 5 is given in Appendix D. From Condition 2, our focus is on only those scenarios where the only possible bargaining solution is $z=0$ and the repeated Game 2 is played just like the static Game 1. From Condition 1 , we further focus on those scenarios where the static Game 1 has poor performance. By using the expression in Proposition 1 , it is easy to verify that for the setting in (31), we have

$$
P_{2}\left(\boldsymbol{y}^{*}(z), z\right)<P_{2}\left(\boldsymbol{y}^{*}(0), 0\right), \quad \forall z>0 .
$$

In this case, we obtain

$$
\mathbb{S}\left(\boldsymbol{y}^{S}, \boldsymbol{z}^{S}, \boldsymbol{v}^{S}\right)=\frac{\left(\gamma_{1}+\gamma_{2}\right)^{2}}{2 a}, \quad \mathbb{S}\left(\boldsymbol{y}^{*}(z=0), z=0\right)=\frac{3 \gamma_{1}^{2}}{8 a} .
$$

Replacing (31) in the above, the efficiency is obtained as $\frac{12}{25}$. Theorem 5 directly leads to the following key result.

Theorem 6: If both users play the grim-trigger strategy, then

$$
\text { PoA }\left(\text { Game 2, Problem 1) } \leq \frac{12}{25}=48 \%\right. \text {. }
$$

The above upper bound holds for any bargaining scheme.

\section{A Simple and EfFicient Bargaining Solution}

In general, finding the Nash bargaining solution in (20) can be difficult due to the complexity of the objective function and the constraints. In this section, we propose a simple bargaining scheme which can be easily implemented among the users, e.g., before they start to perform inter-session network coding. 


\section{A. Min-Max Bargaining Solution}

The key idea in min-max bargaining scheme is to let each user individually make a choice for the coding rate $z$ and then the bargaining solution is selected between the two choices such that both (14) and (15) hold, i.e., both users benefit from network coding. Consider the following set for user 1 :

$$
\begin{aligned}
\mathcal{Z}_{1}=\{ & z \geq 0 \mid \forall \hat{z} \in[0, z], \\
& \left.P_{1}\left(\boldsymbol{y}^{*}(z), z\right) \geq P_{1}\left(\boldsymbol{y}^{*}(\hat{z}), \hat{z}\right) \geq P_{1}\left(\boldsymbol{y}^{*}(0), 0\right)\right\} .
\end{aligned}
$$

We note that user 1's payoff $P_{1}\left(\boldsymbol{y}^{*}(z), z\right)$ is monotonically increasing over set $\mathcal{Z}_{1}$. Furthermore, any $z \in \mathcal{Z}_{1}$ satisfies condition (14), making it an acceptable coding rate as far as user 1 is concerned. Similarly, for user 2 , we define a set

$$
\begin{aligned}
\mathcal{Z}_{2}=\{ & \{z \geq 0 \mid \forall \hat{z} \in[0, z], \\
& \left.P_{2}\left(\boldsymbol{y}^{*}(z), z\right) \geq P_{2}\left(\boldsymbol{y}^{*}(\hat{z}), \hat{z}\right) \geq P_{2}\left(\boldsymbol{y}^{*}(0), 0\right)\right\} .
\end{aligned}
$$

From (34) and (35), payoffs $P_{1}\left(\boldsymbol{y}^{*}(z), z\right)$ and $P_{2}\left(\boldsymbol{y}^{*}(z), z\right)$ are increasing over set $\mathcal{Z}_{1} \cap \mathcal{Z}_{2}$. Thus, any choice of $z \in \mathcal{Z}_{1} \cap \mathcal{Z}_{2}$ satisfies both (14) and (15) and can be considered as a potential bargaining solution. Thus, from Theorem 3, we further have

Corollary 2: The grim-trigger strategy in Definition 8 is a subgame perfect equilibrium for Game 2 if we select $z \in$ $\mathcal{Z}_{1} \cap \mathcal{Z}_{2}$ and a discount factor $\delta \geq \delta_{\min }$ for some $\delta_{\min } \in(0,1]$.

We are now ready to make a formal definition as follows.

Definition 9 (Min-Max Bargaining): The min-max bargaining solution is obtained by solving the following problem:

$$
\begin{aligned}
\underset{z \in \mathcal{Z}_{1} \cap \mathcal{Z}_{2}}{\operatorname{maximize}} & \left(P_{1}\left(\boldsymbol{y}^{*}(z), z\right)-P_{1}\left(\boldsymbol{y}^{*}(0), 0\right)\right) \\
& \times\left(P_{2}\left(\boldsymbol{y}^{*}(z), z\right)-P_{2}\left(\boldsymbol{y}^{*}(0), 0\right)\right) .
\end{aligned}
$$

Problems (20) and (36) have the same objective functions. However, in (36), we restrict the feasible set to $\mathcal{Z}_{1} \cap \mathcal{Z}_{2}$. Problem (36) is easier to solve than the Nash bargaining problem in (20). Due to the monotonicity of the objective function in (36) over set $\mathcal{Z}_{1} \cap \mathcal{Z}_{2}$, the optimal solution becomes

$$
z^{*}=\operatorname{maximize}_{z \in \mathcal{Z}_{1} \cap \mathcal{Z}_{2}} z
$$

That is, the min-max bargaining solution $z^{*}$ is the largest member in set $z \in \mathcal{Z}_{1} \cap \mathcal{Z}_{2}$. We can further show that

$$
z^{*}=\min \left\{z_{1}^{*}, z_{2}^{*}\right\},
$$

where $z_{1}^{*}=\max _{z \in \mathcal{Z}_{1}} z$ and $z_{2}^{*}=\max _{z \in \mathcal{Z}_{2}} z$. Interestingly, $z_{1}^{*}$ and $z_{2}^{*}$ are the solutions for selfish problems (18) and (19) as long as these problems are convex. Otherwise, $z_{1}^{*}$ and $z_{2}^{*}$ are the smallest local maximizers of (18) and (19), respectively.

If $z_{1}^{*}>z_{2}^{*}$, then user 1 prefers a higher network coding rate than user 2; however, due to Theorem 2, user 1 is worse off by selecting $v_{1}^{k}=z_{1}^{k}>z_{2}^{*}$ at any stage $k \geq 1$. A similar statement is true for user 2 . Thus, the network coding users 1 and 2 can agree on rate $z=z^{*}$ distributively, after they individually announce $z_{1}^{*}$ and $z_{2}^{*}$, respectively. Given $z=z^{*}$, the users can then play according to the grim-trigger strategy.

\section{B. Numerical Results on Efficiency and Price-of-Anarchy}

Next, we evaluate the min-max bargaining scheme for various choices of parameters. Numerical results for 100 random scenarios are shown in Fig. 2. In each scenario, the link cost

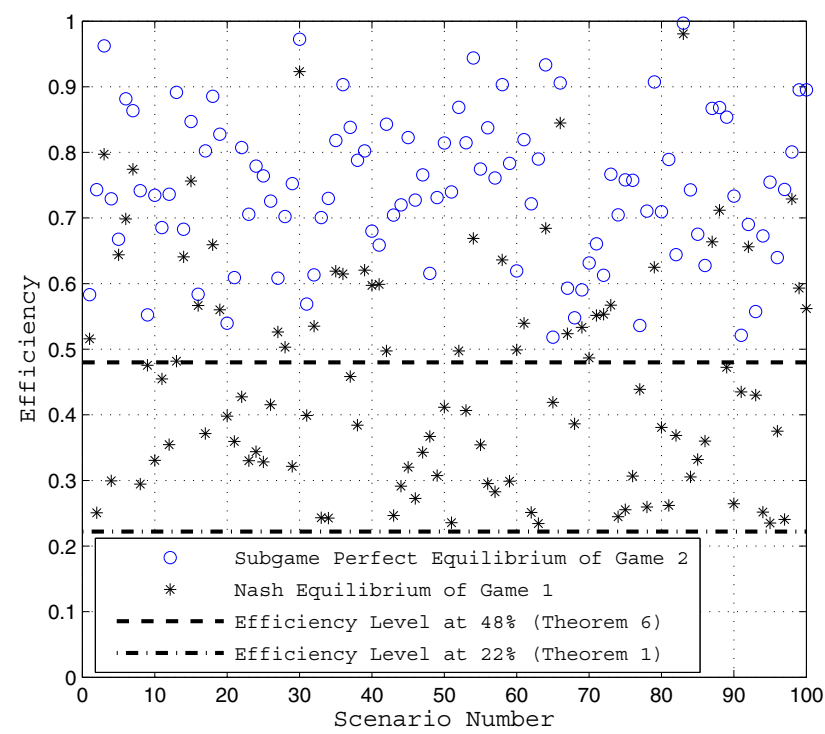

Fig. 2. Efficiency at Nash equilibrium of Game 1 and the subgame perfect equilibrium of Game 2 for 100 random scenarios when the network topology is as in Fig. 1 and the min-max bargaining solution is used. We can see that all circle points appear above the $48 \%$ efficiency level stated in Theorem 6.

parameters $a \in(0,10), b_{1} \in(0,5)$, and $b_{2} \in(0,5)$ are chosen randomly and the utilities functions are $\alpha$-fair [20]:

$$
U_{n}(x)=\gamma_{n}\left(1-\alpha_{n}\right)^{-1} x^{1-\alpha_{n}}, \quad n \in \mathcal{N},
$$

where $\alpha_{n} \in[0,1)$ and $\gamma_{n}>0$ are selected randomly. We can verify that the utility functions in (39) satisfy Assumption 2. In particular, they include the linear case in (25) when $\alpha_{n}=0$.

From Fig. 2, Game 2 has a higher efficiency than Game 1 in every scenario. Moreover, the efficiency of Game 2 is always greater than or equal to $48 \%$, suggesting that PoA (Game 2, Problem 1) $\approx 48 \%$. From this, together with the upper bound result in Theorem 6 , we can conclude that the worst-case efficiency of Game 2 occurs when even bargaining cannot help to encourage users to perform network coding.

\section{CONCLUSION}

This work represents a first step towards understanding non-cooperative inter-session network coding in a repeated game theoretic framework. We focus our study on a simple butterfly network with two network coding users. We show that if the inter-session network coding game is likely to be played repeatedly, then it is possible for the pair of network coding users to achieve a mutually desirable non-zero network coding rate via bargaining. This is in sharp contrast to the static (i.e., played one time only) inter-session network coding games, where no network coding is performed at Nash equilibrium. We also investigate the price-of-anarchy (PoA), i.e., the worst-case efficiency compared to an optimal and cooperative network design. We show that for all possible bargaining schemes, the PoA of the repeated network coding game is upper-bounded by $48 \%$. Furthermore, this upper bound can be achieved if a simple bargaining solution, called min-max bargaining, is used. This indicates a noticeable improvement compared to the $22 \%$ PoA results for static inter-session network coding for the same setting in the literature. 


\section{APPENDIX}

\section{A. Proof of Theorem 2}

Let $\Delta=z_{1}^{k}-z_{2}^{k}>0$. In this case, we have

$$
\begin{aligned}
P_{1} & \left(\overline{\boldsymbol{y}}^{k}, \overline{\boldsymbol{z}}^{k}, \overline{\boldsymbol{v}}^{k}\right)=U_{1}\left(y_{1}^{k}+\Delta+v_{2}^{k}\right)-\left(v_{1}^{k}-\Delta\right) p_{1}\left(v_{1}^{k}-\Delta\right) \\
- & \left(y_{1}^{k}+\Delta+z_{1}^{k}-\Delta-(1-\beta) z_{2}^{k}\right) p\left(y_{1}+y_{2}+\Delta+z_{1}^{k}-\Delta\right) \\
= & U_{1}\left(y_{1}^{k}+\Delta+v_{2}^{k}\right)+\Delta p_{1}\left(v_{1}^{k}-\Delta\right)-v_{1}^{k} p_{1}\left(v_{1}^{k}-\Delta\right) \\
& -\left(y_{1}^{k}+z_{1}^{k}-(1-\beta) z_{2}^{k}\right) p\left(y_{1}+y_{2}+z_{1}^{k}\right) \\
> & U_{1}\left(y_{1}^{k}+v_{2}^{k}\right)-v_{1}^{k} p_{1}\left(v_{1}^{k}-\Delta\right) \\
& -\left(y_{1}^{k}+z_{1}^{k}-(1-\beta) z_{2}^{k}\right) p\left(y_{1}+y_{2}+z_{1}^{k}\right) \\
= & P_{1}\left(\boldsymbol{y}^{k}, \boldsymbol{z}^{k}, \boldsymbol{v}^{k}\right),
\end{aligned}
$$

where the inequality is due to $\Delta p_{1}\left(v_{1}^{k}-\Delta\right)>0, y_{1}^{k}+\Delta+$ $v_{2}^{k}>y_{1}^{k}+v_{2}^{k}$, and the fact that utility function $U_{1}(\cdot)$ is increasing. We can also show that $P_{2}\left(\overline{\boldsymbol{y}}^{k}, \overline{\boldsymbol{z}}^{k}, \overline{\boldsymbol{v}}^{k}\right)=P_{2}\left(\boldsymbol{y}^{k}, \boldsymbol{z}^{k}, \boldsymbol{v}^{k}\right)$. Therefore, user 1 is better off by switching to new rates $\left(\bar{y}_{1}^{k}, \bar{v}_{1}^{k}, \bar{z}_{1}^{k}\right)$, without changing user 2's payoff.

\section{B. Proof of Theorem 3}

First, we prove that the grim-trigger strategy is a subgame perfect equilibrium for user 1 . Assume that both users always cooperate and play Step 1 in Definition 8 . At each stage $k \geq 1$ of Game 2, if user 1 follows the grim-trigger strategy and sets its rates as in Step 1, it expects a long-term payoff

$$
\sum_{t=k}^{\infty}(\delta)^{t-k} P_{1}\left(\boldsymbol{y}^{*}(z), z\right)=\left(P_{1}\left(\boldsymbol{y}^{*}(z), z\right)\right) /(1-\delta) .
$$

On the other hand, assume that user 1 can reach the best payoff $\Gamma_{1} \geq P_{1}\left(\boldsymbol{y}^{*}(z), z\right)$ at the current stage of Game 2 if it deviates from Step 1. Then, user 1 expects a long-term payoff

$$
\begin{aligned}
& \Gamma_{1}+\sum_{t=k+1}^{\infty}(\delta)^{t-k} P_{1}\left(\boldsymbol{y}^{*}(0), 0\right) \\
& =\Gamma_{1}+(\delta /(1-\delta)) P_{1}\left(\boldsymbol{y}^{*}(0), 0\right)
\end{aligned}
$$

Comparing (40) and (41), it is best for user 1 to cooperate if and only if there exists discount factor $\delta \in(0,1]$ such that

$$
\frac{P_{1}\left(\boldsymbol{y}^{*}(z), z\right)}{1-\delta} \geq \Gamma_{1}+\left(\frac{\delta}{1-\delta}\right) P_{1}\left(\boldsymbol{y}^{*}(0), 0\right) .
$$

After reordering the terms, it is required that

$$
1 \geq \delta \geq \frac{\Gamma_{1}-P_{1}\left(\boldsymbol{y}^{*}(z), z\right)}{\Gamma_{1}-P_{1}\left(\boldsymbol{y}^{*}(0), 0\right)}
$$

A similar statement can be shown for user 2. Therefore, for the grim-trigger strategy to form a subgame perfect equilibrium, the discount factor $\delta$ needs to satisfy $\delta \geq \delta_{\min }$, where

$$
\delta_{\min }=\max \left\{\frac{\Gamma_{1}-P_{1}\left(\boldsymbol{y}^{*}(z), z\right)}{\Gamma_{1}-P_{1}\left(\boldsymbol{y}^{*}(0), 0\right)}, \frac{\Gamma_{2}-P_{2}\left(\boldsymbol{y}^{*}(z), z\right)}{\Gamma_{2}-P_{2}\left(\boldsymbol{y}^{*}(0), 0\right)}\right\} .
$$

Here, $\Gamma_{2} \geq P_{2}\left(\boldsymbol{y}^{*}(z), z\right)$ is the best payoff user 2 can achieve in the current stage of Game 2 if it deviates from the rates in Step 1 of the grim-trigger strategy.

\section{Proof of Proposition 1}

Replacing (25) and (26) in Lemma 1, we can consider three cases separately. Here we notice that $q^{*}(z)=0$.

Case I) If $y_{1}^{*}(z)>0$ and $y_{2}^{*}(z)>0$, then

$$
\begin{aligned}
& \gamma_{1}=a\left(y_{1}^{*}(z)+y_{2}^{*}(z)+(1+\beta) z\right)+a y_{1}^{*}(z), \\
& \gamma_{2}=a\left(y_{1}^{*}(z)+y_{2}^{*}(z)+(1+\beta) z\right)+a y_{2}^{*}(z) .
\end{aligned}
$$

From (44), we have $y_{1}^{*}(z)=\frac{\gamma_{1}-a\left(y_{2}^{*}(z)+(1+\beta) z\right)}{2 a}$. Replacing this in (45), we can further show that

$$
\begin{aligned}
& y_{1}^{*}(z)=\left(2 \gamma_{1}-\gamma_{2}-a(1+\beta) z\right) /(3 a), \\
& y_{2}^{*}(z)=\left(2 \gamma_{2}-\gamma_{1}-a(1+\beta) z\right) /(3 a) .
\end{aligned}
$$

From (46) and knowing that $y_{1}^{*}(z)>0$, we have

$$
2 \gamma_{1}-\gamma_{2}-a(1+\beta) z>0 \Rightarrow \quad z<\frac{2 \gamma_{1}-\gamma_{2}}{a(1+\beta)} .
$$

Similarly, from (47) and knowing that $y_{2}^{*}(z)>0$, we have

$$
2 \gamma_{2}-\gamma_{1}-a(1+\beta) z>0 \Rightarrow z<\frac{2 \gamma_{2}-\gamma_{1}}{a(1+\beta)} .
$$

Since $\gamma_{1} \geq \gamma_{2}$, the inequalities in (48) and (49) reduce to

$$
0 \leq z<\left(2 \gamma_{2}-\gamma_{1}\right) / a(1+\beta) .
$$

Thus, the data rates in (46) and (47) hold only if (50) holds.

Case II) If $y_{1}^{*}(z)>0$ and $y_{2}^{*}(z)=0$, then

$$
\begin{aligned}
& \gamma_{1}=a\left(y_{1}^{*}(z)+(1+\beta) z\right)+a y_{1}^{*}(z), \\
& \gamma_{2} \leq a\left(y_{1}^{*}(z)+(1+\beta) z\right) .
\end{aligned}
$$

From (51) and after reordering the terms, we have

$$
y_{1}^{*}(z)=\left(\gamma_{1}-a(1+\beta) z\right) /(2 a) .
$$

Replacing (53) in (52), we have

$$
2 \gamma_{2} \leq \gamma_{1}+a(1+\beta) z \quad \Rightarrow \quad z \geq\left(2 \gamma_{2}-\gamma_{1}\right) /(a(1+\beta)) .
$$

Moreover, from (53) and knowing that $y_{1}^{*}(z)>0$, we have $\gamma_{1}>a(1+\beta) z$. From this, together with (54), we have

$$
\left(2 \gamma_{2}-\gamma_{1}\right) /(a(1+\beta)) \leq z<\gamma_{1} /(a(1+\beta)) .
$$

Case III) If $y_{1}^{*}(z)=0$ and $y_{2}^{*}(z)=0$, then

$$
\gamma_{1} \leq a(1+\beta) z, \quad \gamma_{2} \leq a(1+\beta) z .
$$

Since $\gamma_{1} \geq \gamma_{2}$, we have $z \geq \frac{\gamma_{1}}{a(1+\beta)}$.

\section{Proof of Theorem 5}

Without loss of generality, we assume that $\gamma_{1} \geq \gamma_{2}$. In that case, given $z \geq 0$, the data rates $y_{1}^{*}(z)$ and $y_{2}^{*}(z)$ are obtained from Proposition 1. We consider three cases separately.

Case I) If $\gamma_{2} \leq \gamma_{1}<2 \gamma_{2}$ and (25) and (26) hold, then

$$
\begin{aligned}
& P_{2}\left(\boldsymbol{y}^{*}(0), 0\right)= \\
& \quad \gamma_{2}\left(\frac{2 \gamma_{2}-\gamma_{1}}{3 a}\right)-a\left(\frac{2 \gamma_{2}-\gamma_{1}}{3 a}\right)\left(\frac{\gamma_{1}+\gamma_{2}}{3 a}\right) .
\end{aligned}
$$

On the other hand, if $0 \leq z<\frac{2 \gamma_{2}-\gamma_{1}}{a(1+\beta)}$ and $\beta=\frac{1}{2}$, then

$$
\begin{aligned}
& P_{2}\left(\boldsymbol{y}^{*}(z), z\right)= \\
& \quad \gamma_{2}\left(\frac{2 \gamma_{2}-\gamma_{1}}{3 a}+\frac{z}{2}\right)-a\left(\frac{2 \gamma_{2}-\gamma_{1}}{3 a}\right)\left(\frac{\gamma_{1}+\gamma_{2}}{3 a}\right) .
\end{aligned}
$$


From (57) and (58), we have

$P_{2}\left(\boldsymbol{y}^{*}(z), z\right)-P_{2}\left(\boldsymbol{y}^{*}(0), 0\right)=\frac{\gamma_{2} z}{2}>0, \forall z \in\left(0, \frac{2 \gamma_{2}-\gamma_{1}}{a(1+\beta)}\right)$.

Therefore, the inequality in (22) may not hold in this case. A similar statement is true for inequality (21). Thus, Condition 1 does not hold if $\gamma_{2} \leq \gamma_{1}<2 \gamma_{2}$.

Case II) If $2 \gamma_{2} \leq \gamma_{1} \leq 4 \gamma_{2}$ and (25) and (26) hold, then

$$
P_{2}\left(\boldsymbol{y}^{*}(0), 0\right)=0 \text {. }
$$

On the other hand, if $0 \leq z<\frac{\gamma_{1}}{a(1+\beta)}$ and $\beta=\frac{1}{2}$, then

$$
P_{2}\left(\boldsymbol{y}^{*}(z), z\right)=\gamma_{2} z-\frac{a z}{2}\left(\frac{\gamma_{1}}{2 a}+\frac{z}{4}\right) \text {. }
$$

Furthermore, we have

$$
\lim _{z \rightarrow 0} \frac{d P_{2}\left(\boldsymbol{y}^{*}(z), z\right)}{d z}=\lim _{z \rightarrow 0} \gamma_{2}-\frac{\gamma_{1}}{4}-\frac{a z}{4}=\gamma_{2}-\frac{\gamma_{1}}{4}>0 .
$$

Therefore, the inequality in (22) may not hold in this case. A similar statement is true for inequality (21). Thus, Condition 1 does not hold if $2 \gamma_{2} \leq \gamma_{1}<4 \gamma_{2}$.

Case III) If $4 \gamma_{2} \leq \gamma_{1}$ and (25) and (26) hold, then

$$
P_{2}\left(\boldsymbol{y}^{*}(0), 0\right)=0 \text {. }
$$

If $0 \leq z<\frac{\gamma_{1}}{a(1+\beta)}$ and $\beta=\frac{1}{2}$, then (60) holds and we have

$$
\begin{aligned}
& P_{2}\left(\boldsymbol{y}^{*}(z), z\right)-P_{2}\left(\boldsymbol{y}^{*}(0), 0\right) \\
& \quad=z\left(\gamma_{2}-\frac{\gamma_{1}}{4}\right)-\frac{a z^{2}}{4}<0, \quad \forall z \in\left(0, \frac{\gamma_{1}}{a(1+\beta)}\right),
\end{aligned}
$$

where the inequality is due to $4 \gamma_{2} \leq \gamma_{1}$. On the other hand, if $\gamma_{1} / a(1+\beta) \leq z$ and $\beta=\frac{1}{2}$, then

$$
P_{2}\left(\boldsymbol{y}^{*}(z), z\right)=\gamma_{2} z-\left(a z^{2}\right) / 2 .
$$

Therefore, we have

$$
\begin{aligned}
& P_{2}\left(\boldsymbol{y}^{*}(z), z\right)-P_{2}\left(\boldsymbol{y}^{*}(0), 0\right) \quad \forall z \geq \frac{\gamma_{1}}{a(1+\beta)}, \\
& \quad=z\left(\gamma_{2}-\frac{a z}{2}\right)<0,
\end{aligned}
$$

where the inequality is due to

$$
\gamma_{2}-\frac{a z}{2}<\gamma_{2}-\frac{a}{2}\left(\frac{\gamma_{1}}{a\left(1+\frac{1}{2}\right)}\right)=\gamma_{2}-\frac{\gamma_{1}}{3}<0 .
$$

From (62) and (65), the inequality (22) holds if and only if

$$
0<\gamma_{2} \leq \frac{\gamma_{1}}{4}
$$

In that case, from Corollary 1 , we have

$$
z=0, \quad y_{1}^{*}(0)=\frac{\gamma_{1}}{2 a}, \quad y_{2}^{*}(0)=0 .
$$

Thus,

$$
\mathbb{S}\left(\boldsymbol{y}^{*}(z=0), z=0\right)=\gamma_{1}\left(\frac{\gamma_{1}}{2 a}\right)-\frac{a}{2}\left(\frac{\gamma_{1}}{2 a}\right)^{2}=\frac{3 \gamma_{1}^{2}}{8 a} .
$$

On the other hand, from [6, Theorem 10], we have

$$
\mathbb{S}\left(\boldsymbol{y}^{S}, \boldsymbol{z}^{S}, \boldsymbol{v}^{S}\right)=\frac{\left(\gamma_{1}+\gamma_{2}\right)^{2}}{2 a}
$$

Therefore, the worst-case efficiency of Game 2 is obtained by solving the following optimization problem

$$
\begin{array}{ll}
\underset{\gamma_{1}, \gamma_{2}, a}{\operatorname{minimize}} & \left(\frac{3 \gamma_{1}^{2}}{8 a}\right) /\left(\frac{\left(\gamma_{1}+\gamma_{2}\right)^{2}}{2 a}\right) \\
\text { subject to } & 0<\gamma_{2} \leq \frac{\gamma_{1}}{4} .
\end{array}
$$

The objective function in (70) is decreasing in $\gamma_{2}$. Thus, the minimum occurs when $\gamma_{2}=\frac{\gamma_{1}}{4}$. The efficiency becomes

$$
\left(\frac{3 \gamma_{1}^{2}}{8 a}\right) /\left(\frac{\left(\gamma_{1}+\frac{\gamma_{1}}{4}\right)^{2}}{2 a}\right)=\left(\frac{3}{8}\right) /\left(\frac{25}{32}\right)=\frac{12}{25} .
$$

We can see that the worst-case efficiency does not depend on the exact value of shared-link cost parameter $a$ and also the exact value of utility parameter $\gamma_{1}$. It only depends on the relative values of utility parameters $\gamma_{1}$ and $\gamma_{2}$.

\section{ACKNOWLEDGMENT}

This work is supported by the Natural Sciences and Engineering Research Council (NSERC) of Canada, the GRF Grants 412308 and 412509 from Hong Kong University Grant Committee, and the National Key Technology R\&D Program under the grant number 2007BAH17B04 from the Ministry of Science and Technology in Hong Kong, China.

\section{REFERENCES}

[1] R. Ahlswede, N. Cai, S. Li, and R. Yeung, "Network information flow," IEEE Trans. on Information Theory, vol. 46, pp. 1204-1216, Apr. 2000.

[2] L. Chen, T. Ho, S. Low, M. Chiang, and J. Doyle, "Optimization based rate control for multicast with network coding," in Proc. of IEEE INFOCOM, Anchorage, AK, May 2007.

[3] D. Traskov, N. Ratnakar, D. S. Lun, R. Koetter, and M. Medard, "Network coding for multiple unicasts: An approach based on linear optimization," in Proc. of IEEE ISIT, Seattle, WA, July 2006.

[4] C. C. Wang and N. B. Shroff, "Beyond the butterfly: Graph-theoretic characterization of the feasibility of network coding with two simple unicast sessions," in Proc. of IEEE ISIT, Nice, France, June 2007.

[5] A. H. Mohsenian Rad, J. Huang, V. Wong, S. Jaggi, and R. Schober, "A game-theoretical analysis of inter-session network coding," in Proc. of IEEE ICC, Dresden, Germany, June 2009.

[6] —, "Inter-session network coding with strategic users: A gametheoretic analysis of network coding," submitted to IEEE Trans. on Information Theory, http://arxiv.org/pdf/0904.2921, Apr. 2009.

[7] J. Price and T. Javidi, "Network coding games with unicast flows," IEEE J. on Selected Areas in Commun., vol. 26, pp. 1302-1316, Sept. 2008.

[8] D. Fudenberg and J. Tirole, Game Theory. MIT Press, 1991.

[9] C. Long, Q. Zhang, B. Li, H. Yang, and X. Guan, "Non-cooperative power control for wireless ad hoc networks with repeated games," IEEE J. on Sel. Areas in Commun., vol. 25, no. 6, pp. 1101-1112, Aug. 2007.

[10] Z. Li, "Cross-monotonic multicast," in Proc. of IEEE INFOCOM, Phoenix, AZ, Apr. 2008.

[11] A. Ramamoorthy, V. Roychowdhury, and S. K. Singh, "Selfish distributed compression over networks: Correlation induces anarchy," submitted to IEEE Trans. on Information Theory, Apr. 2009.

[12] J. R. Marden and M. Effros, "The price of selfishness in network coding," in Proc. of 5th Workshop on Network Coding Theory and Applications, Lausanne, Switzerland, June 2009.

[13] T. Ho, Y. Chang, and K. Han, "On constructive network coding for multiple unicasts," in Proc. of Annual Allerton Conference on Communications, Control, and Computing, Urbana, IL, Sept. 2006.

[14] F. P. Kelly, A. Maulloo, and D. Tan, "Rate control for communication networks: Shadow prices, proportional fairness and stability," Journal of Operations Research Society, vol. 49, pp. 237-252, Mar. 1998.

[15] D. Samet and Y. Tauman, "The determination of marginal cost prices under a set of axioms," Econometrica, vol. 59, pp. 895-909, 1982.

[16] R. Johari and J. N. Tsitsiklis, "A scalable network resource allocation mechanism with bounded efficiency loss," IEEE J. on Selected Areas in Commun., vol. 24, no. 5, pp. 992-999, May 2006.

[17] S. Yang and B. Hajek, "VCG-Kelly mechanisms for allocation of divisible goods: Adapting VCG mechanisms to one-dimensional signals," in Proc. of CISS, Princeton, NJ, Mar. 2006.

[18] T. Driessen, Cooperative Games, Solutions and Applications. Kluwer Academic Publishers, 1988.

[19] A. E. Roth, Axiomatic Models of Bargaining: Lecture Notes in Economics and Mathematical Systems. Springer-Verlag, 1990.

[20] J. Mo and J. Walrand, "Fair end-to-end window-based congestion control," IEEE/ACM Trans. on Net., vol. 8, pp. 556-567, Oct. 2000. 